\title{
Polygonal Eyewall and Mesovortices Structure in a Numerically Simulated Typhoon Rusa
}

\author{
Wataru Mashiko \\ Meteorological Research Institute, Tsukuba, Japan
}

\begin{abstract}
In this study, to elucidate the inner structure of Typhoon Rusa (2002), a numerical simulation was conducted using a two-way triple-nested cloud-resolving nonhydrostatic model with a $2 \mathrm{~km}$ horizontal grid size on the finest nested mesh. The model successfully reproduced the features of polygonal eyewall structure observed in Typhoon Rusa. The simulated asymmetric structure in the inner-core region was dominated by a number of mesovortices within or near the eyewall in the lower and middle troposphere. Meso-lows on the horizontal scale of $20-30 \mathrm{~km}$ were found at the kinks of polygonal eyewall, and between them meso-highs existed. The modification of radial flow by the mesovortices affected not only the location of eyewall, but also the convective activities in the eyewall by causing the interaction between the eye and the eyewall. The horizontal distribution of the potential vorticity (PV) showed the wave-like pattern related to the mesovortices near the eyewall, which was quite similar to the ideal numerical experiments of Schubert et al. (1999) and Kossin and Schubert (2001).
\end{abstract}

\section{Introduction}

Due to the advance of observational technology, the detailed asymmetric structure in tropical cyclone cores is often captured. For example, the visible images from a polar-orbiting satellite revealed a remarkable pentagonal eye pattern with mesovortices in Hurricane Isabel (2003) (Kossin and Schubert 2004). Mesovortices around the eyewall cause severe weather such as torrential rainfall and hazardous winds (Black and Marks 1991), and therefore understanding the details of their structure is important to prevent disasters.

The studies of the asymmetry in tropical cyclone cores have been mainly conducted within a theoretical framework of unforced two-dimensional barotropic flow. Kuo et al. (1999) attributed the elliptical eye of Typhoon Herb (1996) to a wavenumber-2 vortex Rossby wave that propagated upwind azimuthally near the eyewall. Schubert et al. (1999) suggested that the polygonal eyewall and mesovortices in hurricanes formed as a result of barotropic instability near the radius of maximum winds, around which the potential vorticity maximum was found. Kossin and Schubert (2001) extended this work, and they suggested that a variety of polygonal shapes showed up due to the merge of the mesovortices around the eyewall and that this process was essential for a hurricane's rapid intensification. However, numerical experiments of tropical cyclones with full-physics and high resolution have rarely been conducted due to the limited computer resources until recent years. Wang (2002a) and Braun (2002) identified vortex Rossby waves in the simulated tropical cyclone

Corresponding author: Meteorological Research Institute, 1-1 Nagamine, Tsukuba 305-0052, Japan. E-mail: wmashiko@mrijma.go.jp. (22005, the Meteorological Society of Japan. core region, and they indicated that the wavenumber- 2 wave was responsible for the generation of elliptical eyewalls. Wang (2002b) suggested that a combination of wavenumber-1 and - 2 waves result in polygonal eyewalls, but it was not shown clearly. Although these recent studies have revealed many characteristics of elliptical eyewalls affected by vortex Rossby waves, mesovortices around the polygonal eyewall have not yet been reproduced by the three dimensional models with full-physics. Therefore, their structures are still poorly understood.

The aim of this paper is to show the successfully simulated mesovortices related to polygonal eyewall shape in Typhoon Rusa and to elucidate their structures and roles by using a three-dimensional cloud-resolving nonhydrostatic model.

\section{Radar observation of Tyhoon Rusa}

Typhoon Rusa passed over the Amami Island in a mature stage with the central minimum pressure of 950 $\mathrm{hPa}$ at 12 UTC on 29 Aug. 2002. The operational JMA (Japan Meteorological Agency) radar located in Naze city on the Amami Island, denoted by $\times$ in Fig. 1, captured the detailed eyewall structure. The distribution of precipitation intensity observed by Naze radar shows a large eye surrounded by the polygonal eyewall pattern with a radius of about $100 \mathrm{~km}$ (Fig. 1). Several straight line segments of band-shaped rainfall areas were often identified. Rusa had a double eye structure, before it came near the Amami Island. The polygonal eyewall corresponded to the outer eyewall, while the inner eyewall collapsed as the Typhoon came near the Amami Island. The straight line segments were divided by several kinks in the eyewall. The remarkable kinks are shown with symbols of A-D in Fig. 1. An interesting feature is that rainfall was strong near most of the kinks and on the azimuthally upwind side of kinks ( $\mathrm{C}$ and $\mathrm{D}$ in Fig. 1), and the inner rainband extended outward from the kinks ( $\mathrm{C}$ in Fig. 1). They slowly rotated anticlockwise around the typhoon center with a period of about 300 minutes.

\section{Brief description of the numerical model}

Numerical experiment of Typhoon Rusa was conducted with a two-way triple-nested movable mesh Typhoon model (Mashiko and Muroi 2003). This model is based on Meteorological Research Institute/Numerical Prediction Division unified nonhydrostatic model of JMA (MRI/NPD-NHM; Saito et al. 2001). Figure 2a shows the model domain and two interior domains that are movable to cover the storm center. The innermost domain $\mathrm{C}$ with a $2 \mathrm{~km}$ horizontal grid size is designed to explicitly resolve the central core of the Typhoon, which covers an horizontal area of $540 \mathrm{~km}$ by $540 \mathrm{~km}$. Grids sizes of $6 \mathrm{~km}$ with a domain of $1500 \mathrm{~km}$ by 1500 $\mathrm{km}$ and $18 \mathrm{~km}$ with a domain of $5400 \mathrm{~km}$ by $3960 \mathrm{~km}$ are used for the intermediate B and outermost domain 


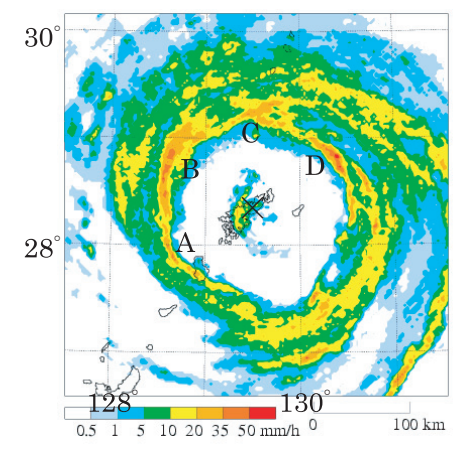

Fig. 1. Distribution of precipitation intensity observed by Naze radar at 1050 UTC on 29 Aug $2002 . \times$ shows radar site. Alphabets (A-D) indicate the remarkable kinks of polygon.

A, respectively. The domain A was one-way nested with the Regional Spectral Model (RSM) (NPD/JMA 2002), an operational hydrostatic model of JMA. The initial field was obtained from the 12-hour forecast of RSM (Fig. 2b). But, the innermost domain $\mathrm{C}$ was initialized at 32-hour forecast by interpolating output from the domain $\mathrm{B}$, and it was integrated for 25 hours. For the model precipitation scheme, cold-rain explicit cloud microphysics is employed and convective adjustment parameterization (Saito et al. 2001) is used conjunctionally only in the domain A.

\section{Numerical results}

\subsection{Overall simulation results of Rusa}

Figure 2a shows the tracks of Typhoon Rusa in the simulation (blue circle) and the analyses of JMA (red box). The simulated typhoon moved westward in comparison with the analyses, and the track error was about $250 \mathrm{~km}$ at $\mathrm{t}=57 \mathrm{~h}$. The central minimum pressure had positive bias of about $12 \mathrm{hPa}$ at the initial time, but it agreed with the analysis $(950 \mathrm{hPa})$ in the later integration period (not shown). The simulated typhoon exhibited a pentagonal eyewall structure around $t=53 \mathrm{~h} 30 \mathrm{~m}$. The general features were reproduced successfully, although the simulated details differed somewhat from the radar analysis (Fig. 1), such as the number of kinks.

\subsection{Characteristics of simulated polygonal eyewall}

Figures $3 a-3 d$ show the vertically accumulated hydrometeors (hereafter $\mathrm{ACH}$ ) of cloud water, cloud ice, rainwater, snow and graupel, the vertical velocity at a height of $2900 \mathrm{~m}$, the horizontal wind velocity at the lowest level of $20 \mathrm{~m}$ height and the relative vorticity at a height of $620 \mathrm{~m}$ at $\mathrm{t}=53 \mathrm{~h} 30 \mathrm{~m}$, respectively. The sea surface pressure field is also shown with contours in Fig. 3. The straight line segments of $\mathrm{ACH}$, especially inner edge of $\mathrm{ACH}$, were divided by several kinks corresponding to those of sea surface pressure. They made a pentagonal-shaped eyewall that was distant about 100 $\mathrm{km}$ from the typhoon center. The distributions of $\mathrm{ACH}$ and upward motion (Fig. $3 \mathrm{a}$ and $3 \mathrm{~b}$ ) show that the convective activities in the eyewall were enhanced from the kinks to their azimuthally upwind side (except at the kink in the west). This feature is similar to that of the observed radar reflectivity, as shown in Fig. 1. The peak of the horizontal wind velocity was found near the kinks and it was about $20 \%$ faster than the averaged azimuthal wind speed (Fig. 3c). The relative vorticity exceeded $3 \times 10^{-3} \mathrm{~s}^{-1}$ there (Fig. 3d). These indicate that the
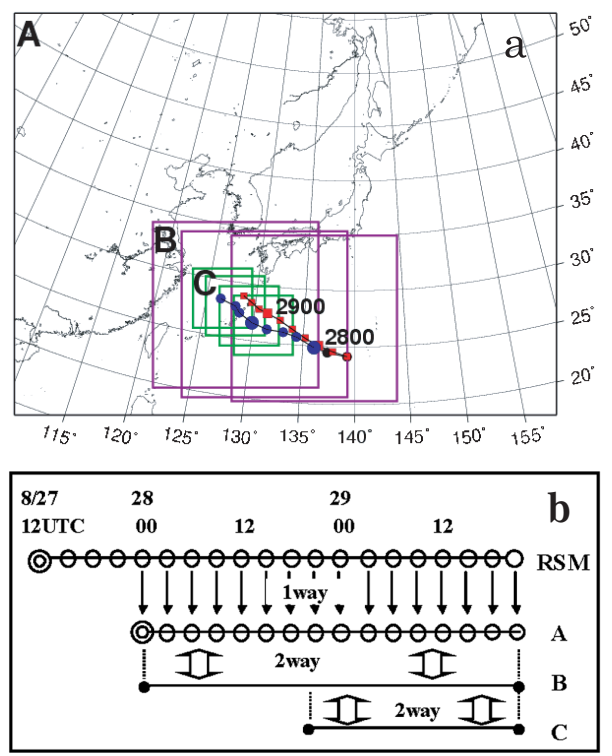

Fig. 2. Design of (a) model domains and (b) relationship of nesting. Tracks of Typhoon Rusa from JMA best track analyses (red box) and the model output (blue circle) every $6 \mathrm{~h}$. Larger fonts denote the positions every $24 \mathrm{~h}$, with the corresponding date and time given (UTC).
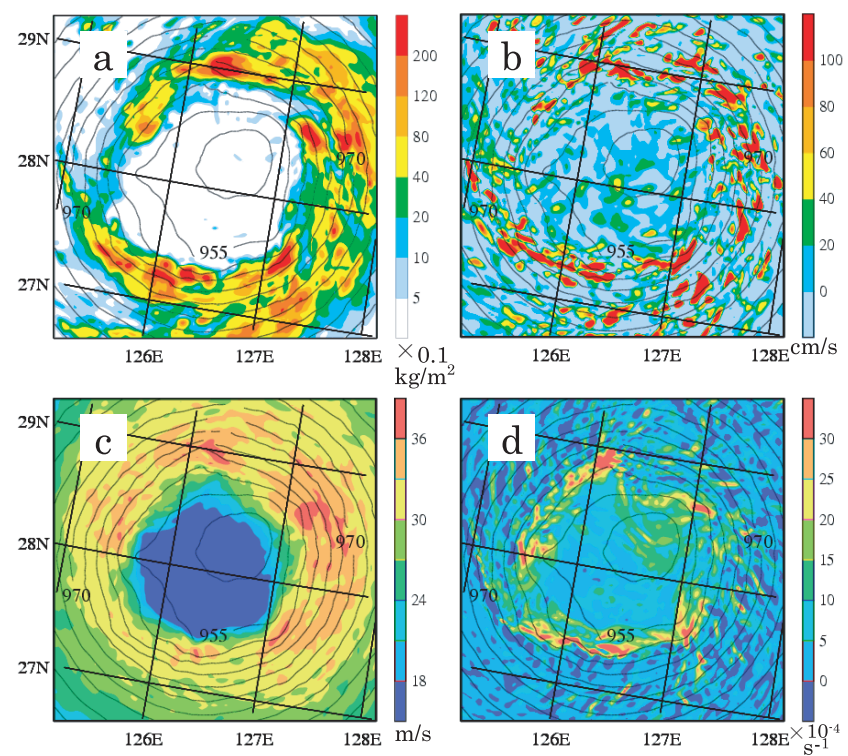

Fig. 3. (a) Vertically accumulated hydrometeors of cloud water, cloud ice, rainwater, snow and graupel, (b) vertical velocity at a height of $2900 \mathrm{~m}$, (c) horizontal wind speed at a lowest level (20 m height) and (d) vertical component of relative vorticity at a height of $620 \mathrm{~m}$ superposed by sea surface pressure with contour (interval; $3 \mathrm{hPa}$ )

enhanced convections near the kinks were accompanied with local wind and vorticity maxima. The pentagonal pattern with these asymmetric features rotated anticlockwise around the center with a period of about 270 minutes. This speed of $39 \mathrm{~m} \mathrm{~s}^{-1}$ was slower than the lower tropospheric tangential wind speed in the eyewall $\left(\sim 50 \mathrm{~m} \mathrm{~s}^{-1}\right)$, and it corresponded to the tangential wind at about a $4-\mathrm{km}$ height. 


\subsection{Structure of the simulated mesovortices}

Figures $4 \mathrm{a}-4 \mathrm{~d}$ show the asymmetric pressure and the asymmetric horizontal wind fields at heights of 620 $\mathrm{m}, 3140 \mathrm{~m}, 5460 \mathrm{~m}$ and $7620 \mathrm{~m}$ at $\mathrm{t}=53 \mathrm{~h} 30 \mathrm{~m}$, respectively. To determine the storm center, a variational approach (Braun 2002) was used on the surface pressure field so that the azimuthal variance at all radii between the center and the outer portion $(120 \mathrm{~km})$ was minimized. The asymmetry around the eyewall was dominated by 5 meso-low pressure perturbations on the horizontal scale of $20-30 \mathrm{~km}$ below about a $4-\mathrm{km}$ height (Figs. 4a and 4b). The meso-lows accompanied with cyclonic flow were located at the kinks of the pentagonal eyewall, and between them 5 meso-high pressure perturbations with anticyclonic flow existed below about a $1.5-\mathrm{km}$ height. The maximum pressure deviation of meso-lows from the azimuthally averaged field was about $3 \mathrm{hPa}$, and that of the meso-highs was $1.5 \mathrm{hPa}$ near the surface (Fig. 4a). The asymmetric structure in the inner area of the eyewall was dominated by wavenumber-1 pattern. The mesovortices structures within or near the eyewall were most predominant near the surface and tilted a little radially outward. The amplitude of the mesovortices weakened with height, and the wavenumber-1 pattern shifted to the upwind side of the azimuthal parent vortex flow dominated widely above a $4-\mathrm{km}$ height. This vertical structure is similar to the observational results of Hurricane Olivia (1994), which revealed that the asymmetry in the inner core was dominated by an azimuthal wavenumber-2 feature below 3-km height and a wavenumber-1 feature above that height (Reasor et al. 2000). The enhanced convective activities in the eastward side (Figs. $3 \mathrm{a}$ and $3 \mathrm{~b}$ ) may be affected by this wavenumber-1 pattern.

The asymmetric wind fields produced the radial outflow between an upstream meso-high and a downstream meso-low, and the radial inflow between an upstream meso-low and a downstream meso-high around the eyewall. The maximum speed of these winds exceeded $10 \mathrm{~m} \mathrm{~s}^{-1}$ in the lower troposphere. They modulated the location of the eyewall, especially the radial outflow region in the southwest side (see Fig. 4a, and Figs. 3a and $3 \mathrm{~b})$.

Besides, these radial flows modified by the mesovortices largely affected the convective activities in the eyewall. As indicated in section 4.2, strong convective activities were found mainly in the azimuthally upwind side of the kinks, which corresponded to the radial outflow region between an upstream meso-high and a downstream meso-low. The area with the high equivalent potential temperature $\theta_{\mathrm{e}}$ extended around the eyewall in the radial outflow region in the lower layer (Fig. 5). Vertical and radial cross sections of $\theta_{\mathrm{e}}$ along the radial inflow (A-C in Fig. 4a) and outflow (C-B in Fig. 4a) are shown in Figs. $6 a$ and $6 \mathrm{~b}$, respectively. The radial outflow was prominent inside the eyewall below a 2 -km height, and it transported the high $\theta_{\mathrm{e}}$ air $(\sim 371 \mathrm{~K})$ from the eye into the eyewall (Fig. 6b). This transportation brought the strong convective activities in the eyewall. Meanwhile, in Fig. 6a the strong near-surface radial inflow reached into the inner eye, $70 \mathrm{~km}$ from the center. Consequently, the convective activities in the eyewall were not so strong. Obviously, however, these relations contradict the general understanding of tropical cyclones that the convective activities in the eyewall are mainly maintained by the frictional inflow in the boundary layer. As mentioned already, Rusa had the extraordinarily large eye. In such the situation, since the dynamical frictional convergence near the eyewall was relatively weak, the strong inflow could not cause strong upward motions in the eyewall.
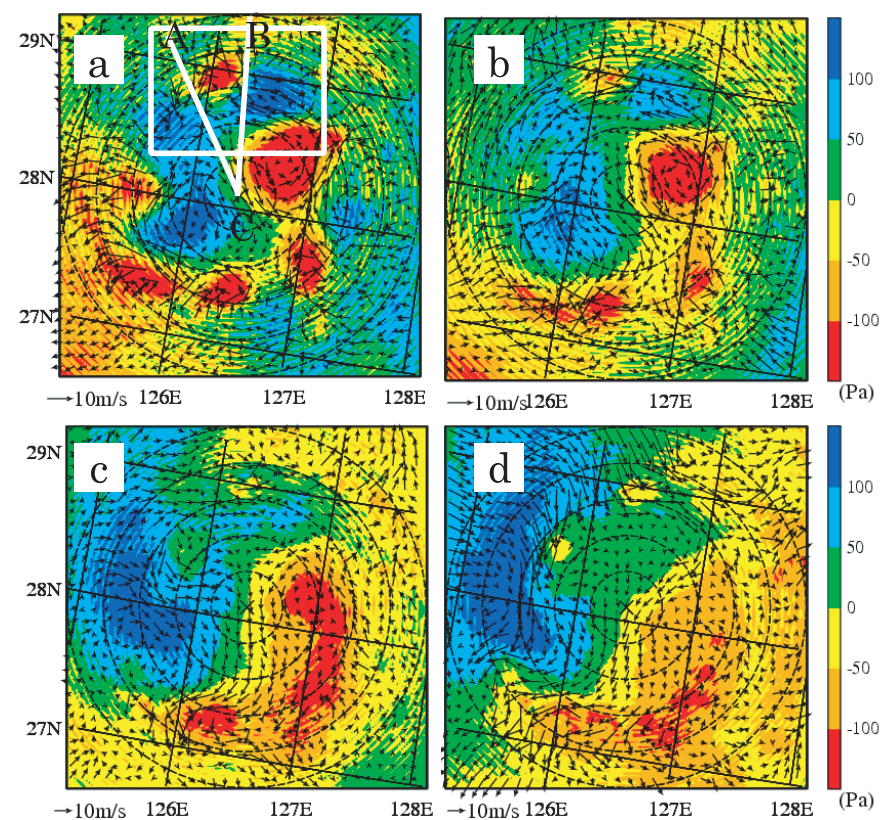

Fig. 4. Shaded in color is the asymmetric pressure field with the asymmetric winds relative to the moving cyclone at a height of (a) $620 \mathrm{~m}$, (b) $3140 \mathrm{~m}$, (c) $5460 \mathrm{~m}$ and (d) $7620 \mathrm{~m}$ after $53 \mathrm{~h} 30 \mathrm{~m}$ of simulation. " $x$ " shows the model typhoon center. The circles are placed at every 30 $\mathrm{km}$ from the center.

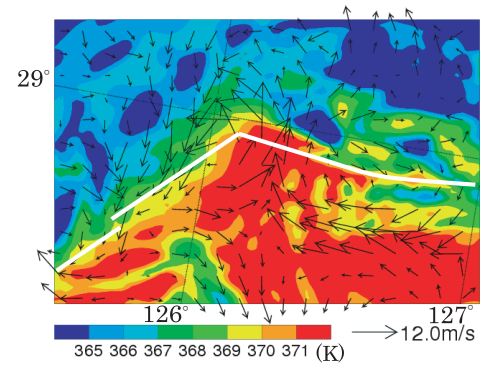

Fig. 5. Horizontal distribution of equivalent potential temperature with asymmetric winds at a height of 620 $\mathrm{m}$ in the square area of Fig. 4a. White solid lines denote the location of the upward motion in the eyewall at the same level.
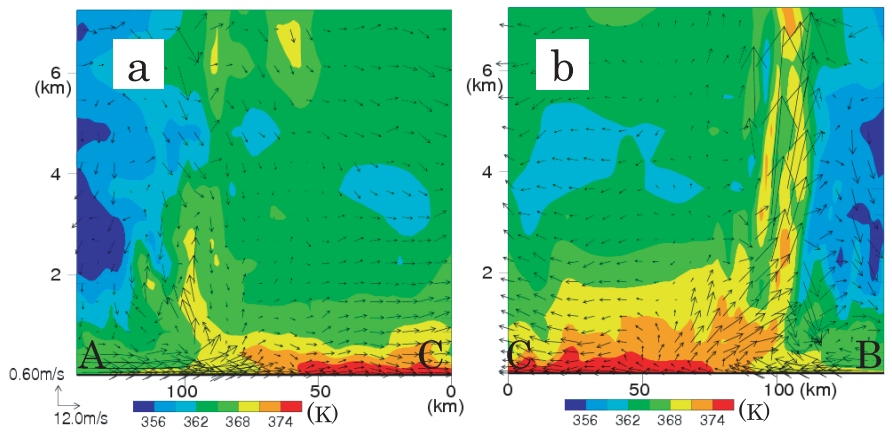

Fig. 6. Vertical cross sections of the simulated equivalent potential temperature superposed by typhoonrelative winds that are taken along (a) line A-C and (b) line C-B in Fig. 4a. 

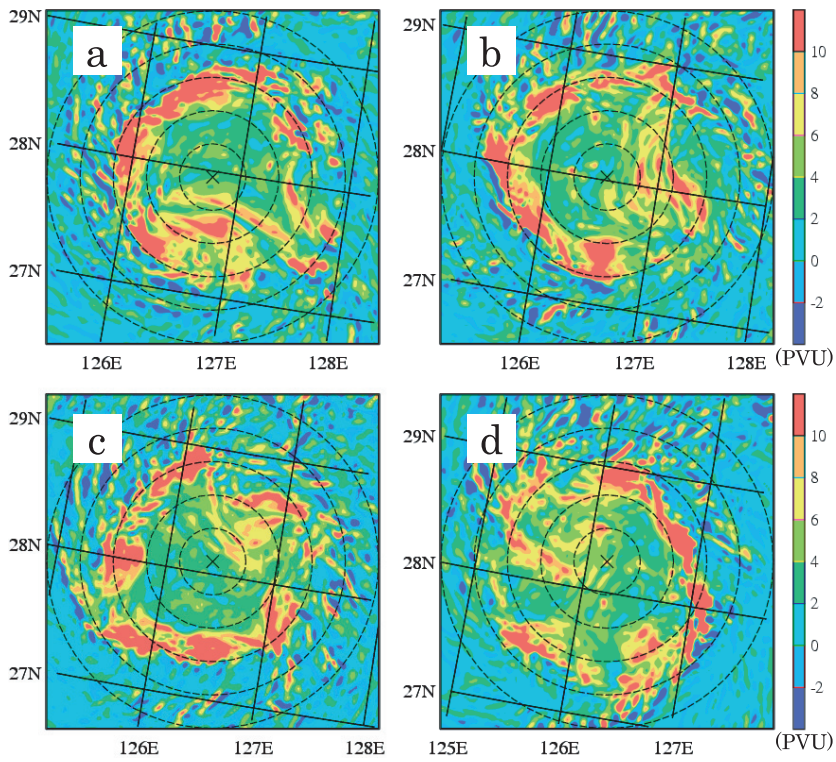

Fig. 7. Model-simulated potential vorticity around the typhoon center at a height of $620 \mathrm{~m}$ from (a) $51 \mathrm{~h} 30 \mathrm{~m}$, (b) $52 \mathrm{~h} 50 \mathrm{~m}$, (c) 53h30m and (d) 55h00m integration. " $\mathrm{x}$ " and the circles are same as in Fig. 4.

\subsection{Evolution of potential vorticity}

Figure 7 shows the evolution of the simulated horizontal distributions of the potential vorticity $(\mathrm{PV})$ at a height of $620 \mathrm{~m}$ during the period of the pentagonal eyewall forming and collapsing (from $t=51 \mathrm{~h} 30 \mathrm{~m}$ to $\mathrm{t}=55 \mathrm{~h} 00 \mathrm{~m})$. The maximum $\mathrm{PV}$ in the eyewall region pooled into 5 discrete parts, and it showed the wave-like pattern (Figs. 7b and $7 \mathrm{c}$ ). These positive PV anomalies accompanied the meso-lows shown in Fig. 4a. After $\mathrm{t}=53 \mathrm{~h} 30 \mathrm{~m}$, the positive PV anomalies collapsed in the westward side, and the PV increased inside of the eyewall. These results are quite similar to Schubert et al. (1999) and Kossin and Schubert (2001). They indicated that the reversal of the radial PV gradient near the eyewall set the stage for dynamic instability and rearrangement of the PV distribution in the barotropic model. In this study, however, strong diabatic heating in the eyewall and surface friction in the boundary layer could play an essential role in the formation and evolution of mesovortices. Therefore, further investigations will be needed to verify how the PV maximum causes dynamic instability and forms several mesovortices. These are in our future study.

\section{Conclusion}

In this study, the polygonal eyewall structure in Typhoon Rusa (2002) was examined by using a twoway triple-nested cloud-resolving nonhydrostatic model with a $2 \mathrm{~km}$ horizontal grid size on the finest nested mesh. The model successfully reproduced the features of the polygonal eyewall structure in the observed Rusa.

Pentagonal eyewall structure was organized by 5 meso-lows and 5 meso-highs within or near the eyewall in the lower and middle troposphere. The meso-lows were found at the kinks of pentagonal eyewall below about a 4-km height, and between them meso-highs existed below about a $1.5-\mathrm{km}$ height. The mesovortices affected not only the eyewall structure but also the convective activities in the eyewall. The radial outflow that was modified by the mesovortices transported the high $\theta_{\mathrm{e}}$ air from the eye into the eyewall, and consequently the convective activities in the eyewall were enhanced there. The horizontal distribution of the PV showed the wave-like pattern near the eyewall, which was quite similar to the ideal numerical experiments of Schubert et al. (1999) and Kossin and Schubert (2001).

This study is the first attempt to reveal the polygonal eyewall and mesovortices structure in a real typhoon by using a three dimensional full-physics model. Further numerical simulations like this study need to ascertain the results of the ideal experiments and theoretical studies. Of course, more detailed observational studies about inner structure of tropical cyclones also need to verify the simulation results.

\section{Acknowledgments}

The author would like to thank M. Ueno, A. Murata, C. Muroi, T. Kato and K. Saito for useful comments and performing simulations. The author also thanks two anonymous reviewers who gave useful comments to improve the manuscript. The numerical experiments are performed using the NEC SX-6 computer system at MRI.

\section{References}

Black, P. G. and F. D. Marks, 1991: The structure of an eyewall meso-vortex in Hurricane Hugo (1989). Preprints, 19th Conf. on Hurricanes and Tropical Meteorology, Miami, FL, Amer. Metor. Soc., 579-582.

Braun, S. A., 2002: A cloud-resolving simulation of Hurricane Bob (1991): Storm structure and eyewall buoyancy. Mon. Wea. Rev., 130, 1573-1592.

Kossin, J. P. and W. H. Schubert, 2001: Mesovortices, polygonal flow patterns, and rapid pressure falls in hurricane-like vortices. I. Atoms. Sci., 58, 2196-2209.

Kossin, J. P. and W. H. Schubert, 2004: Mesovortices in Hurricane Isabel. Bull. Amer. Meteor. Soc., 85, 151-153.

Kuo, H. C., R. T. Williams and J.-H. Chen, 1999: A possible mechanism for the eye rotation of Typhoon Herb. $J$. Atoms. Sci., 56, 1659-1673.

Mashiko, W. and C. Muroi, 2003: Development of a two-way multiply-nested movable mesh typhoon model using the cloud resolving nonhydrostatic model. CAS/JSC WGNE Res. Activities in Atm. and Oceanic Modelling, No.33, 5.225.23 .

Numerical Prediction Division/Japan Meteorological Agency, 2002: Outline of the operational numerical weather prediction of the Japan Meteorological Agency. 158pp.

Reasor, P. D., M. T. Montgomery, F. D. Marks, Jr. and J. F. Gamache, 2000: Low-wavenumber structure and evolution of the hurricane inner core observed by airborne dual-Doppler rader. Mon. Wea. Rev., 128, 1653-1680.

Saito, K., T. Kato, H. Eito and C. Muroi, 2001: Documentation of the Meteorological Research Institute/Numerical Prediction Division Unified Nonhydrostatic Model. Technical Reports of the MRI, 42, $133 \mathrm{pp}$

Schubert, W. H., M. T. Montgomery, R. K. Taft, T. A. Guinn, S. R. Fulton, J. P. Kossin and J. P. Edwards, 1999: Polygonal eyewalls, asymmetric eye contraction, and potential vorticity mixing in hurricanes. J. Atoms. Sci., 56, 11971223.

Wang, Y., 2002a: Vortex Rossby waves in a numerically simulated tropical cyclone. Part I: Overall structure, potential vorticity, and kinetic energy budgets. J. Atoms. Sci., 59, $1213-1238$.

Wang, Y., 2002b: Vortex Rossby waves in a numerically simulated tropical cyclone. Part II: The role in tropical cyclone structure and intensity changes. J. Atoms. Sci., 59, 12391262.

(Manuscript received 6 December 2004, accepted 25 January 2005) 